\title{
Research on Greenhouse Wireless Monitoring System
}

\author{
Xiuqing Wang ${ }^{1, \mathrm{a}}$, Qing Liu ${ }^{1, \mathrm{~b}}$ and Jimin Zhao ${ }^{1, \mathrm{c}}$ \\ ${ }^{1}$ College of Electronic Information and Automation, Tianjin University of Science \& Technology, 1038 \\ Dagu Nanlu, Hexi District, Tianjin 300222, China \\ âxxqly@163.com, ${ }^{\mathrm{a}}$ liuqchn@126.com, ${ }^{\mathrm{c}}$ zhaojiminemail@tust.edu.cn
}

\section{Keywords: Facility agriculture; Acoustic emission; Wireless sensor networks}

Abstract. A greenhouse monitoring system based on wireless sensor network is constructed to collect and transmit the greenhouse environment parameters and acoustic emission (AE) signals under crop disease stress. Low power consumption single chip ATmega8 and sensors for environmental parameters are used to monitor temperature, humidity, carbon dioxide concentration and light intensity; TMS320F28335 processor and fiber Bragg grating (FBG) sensor are chosen to monitor the AE signals under crop disease stress. The upper computer software designed based on LabVIEW displays, analyzes and processes the collected data. The system has strong portability and can be widely used in facility agriculture.

\section{Introduction}

At present, greenhouse mostly focuses on monitoring environmental factors, but there have been few reports on monitoring crop physiological information. It has been found that crop AE signal are a sensitive response of crop to water status and disease, and it can provide important basis for greenhouse regulation [1,2]. Wireless sensor network technology provides a new approach to information acquisition and processing, its application in the greenhouse is conducive to improve the degree of automation of greenhouse and the level of modern management, playing an important role in crop growth monitoring [3]. However, there have been not many researches on the wireless transmission of AE signals [4]. The wired transmission mode will increase the wiring, power supply, maintenance and other costs, which is not suitable for promotion in greenhouses. Therefore, to realize the acquisition and transmission of greenhouse environment parameters and AE signals under crop disease stress, we construct the monitoring system based on wireless sensor network.

\section{Greenhouse Wireless Monitoring System}

In this work, a greenhouse wireless monitoring system is proposed, as shown in Figure 1, the system is divided into data acquisition layer and upper computer monitoring layer. The data acquisition layer uses the environmental parameters sensors to detect the temperature, humidity, carbon dioxide concentration and light intensity of the greenhouse, and transmits the environmental data to the host computer through the wireless network. The AE sensor node is used to detect the AE signals under crop disease stress and transmits the data to the host computer. The upper computer monitoring layer carries out real-time waveform display, analysis, processing and storage of environmental parameters and AE data, based on LabVIEW.

According to the actual requirements of greenhouse system, we employ ZigBee technology to build a greenhouse wireless sensor network monitoring system. The system collects real-time environmental data of temperature, humidity, carbon dioxide concentration, light intensity in the greenhouse, and monitors the AE signals under crop disease stress. For the first four kinds of environmental factors, this paper chooses the single chip microcomputer to process, and then the data is packaged and sent to the DSP through the serial port. Considering the high frequency characteristic of AE signals and the powerful data processing function of DSP, this paper uses DSP as the processing chip of AE signals. The overall structural diagram of the system is shown in figure 2 . 


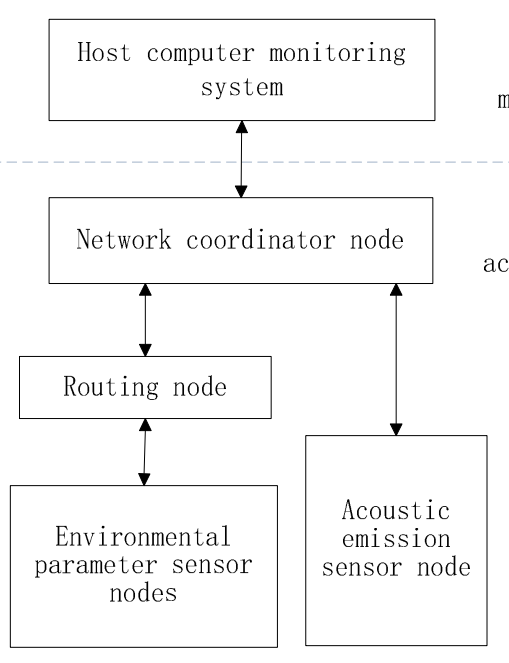

Fig.1 System frame diagram

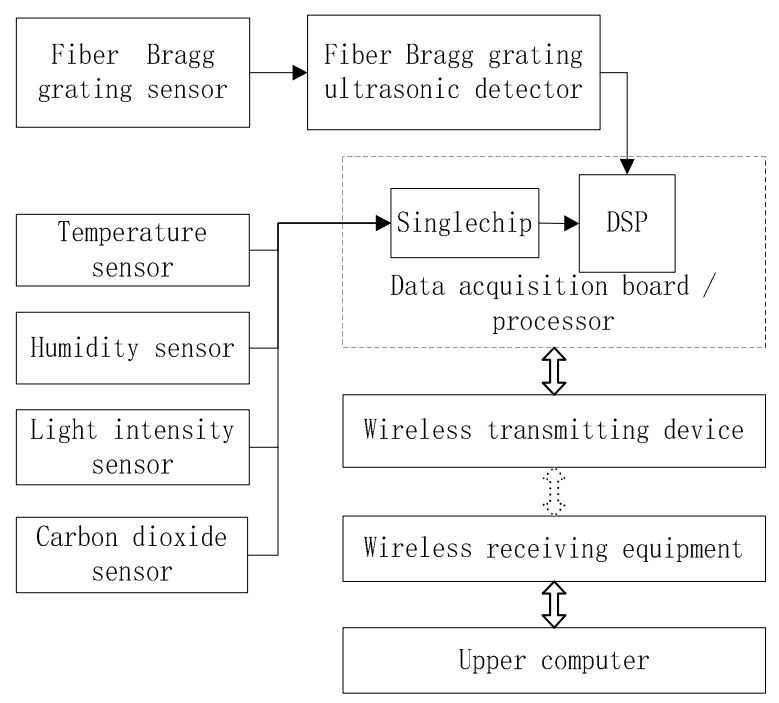

Fig.2 Structural diagram of greenhouse monitoring system

\section{Environmental Parameters Collection}

The wireless sensor network system based on ZigBee is used for the collection and transmission of greenhouse environmental parameters. Wireless sensor network nodes are divided into network coordinator nodes, routing nodes and sensor nodes, modular design is adopted, each node uses a ATmega8 MCU as the processor module to complete AD conversion, preliminary data processing, data reading and other functions, uses SZ05 wireless communication module to complete the wireless transmission and exchange of information. The hardware structure of the sensor node is as shown in figure 3.

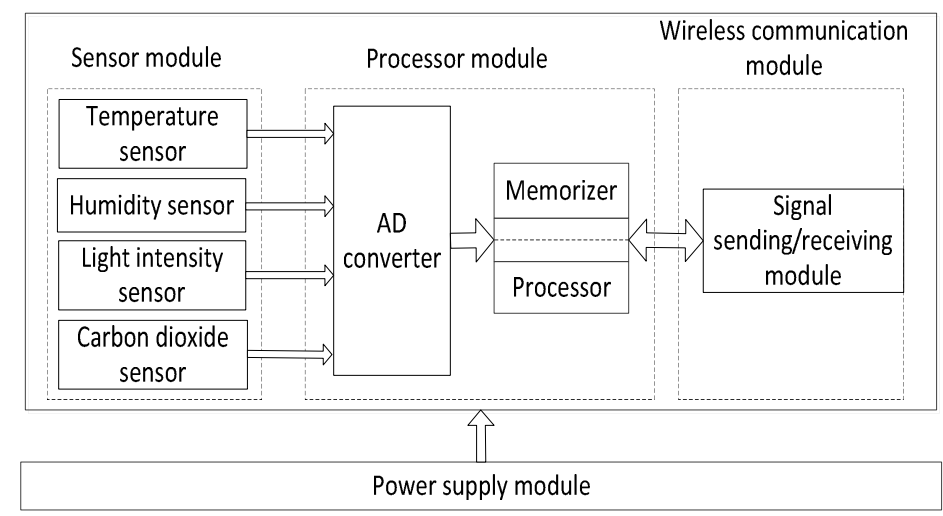

Fig. 3 Hardware structural of the sensor node

In view of the greenhouse monitoring environment, this system builds a tree network through multiple terminal sensor nodes, routing nodes and network coordinator node, and realizes wireless transmission of multi-channel signals. The network coordinator node is responsible for the formation of the whole network in wireless network, and completes the functions of network settings, network management, data receiving and delivering and so on. It is connected with the computer through the serial port. The sensor nodes are arranged in the designated monitoring points of the greenhouse in advance, and regularly transmit collected data to the network coordinator node. The sensor nodes receive and respond to the commands of the network coordinator node at the same time.

\section{Crop AE Signals Acquisition}

The traditional methods of crop disease classification and recognition mainly depend on the human eye observation, which is backward in prediction. It has been found that daily AE events of healthy tomato plants have a certain regularity. However, the regularity of $\mathrm{AE}$ events was distorted under 
disease stress [2]. The crop AE can be observed by continuous monitoring, which can find out the rules of $\mathrm{AE}$ events under diseases stress, to diagnose disease and formulate strategies for prevention and control. It plays an active role in greenhouse regulation.

In recent years, an AE sensor based on FBG has been developed, which has more advantages than traditional piezoelectric sensors, featured by strong anti-electromagnetic interference capacity, corrosion resistance, light weight, small size, etc. and is widely applied. Especially, as for fiber grating AE detection system, because of the FBG sensor itself physical characteristics, there is no AE wave oscillation in the sensor. The waveform acquisition will not produce distortion, which is better than piezoelectric ceramic AE sensing system [5].

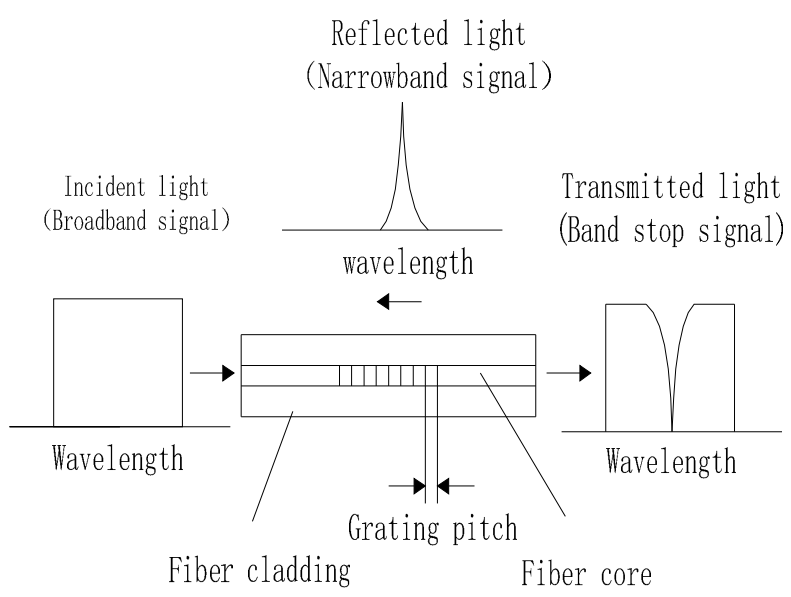

Fig.4 Diagram showing how light propagates through a FBG

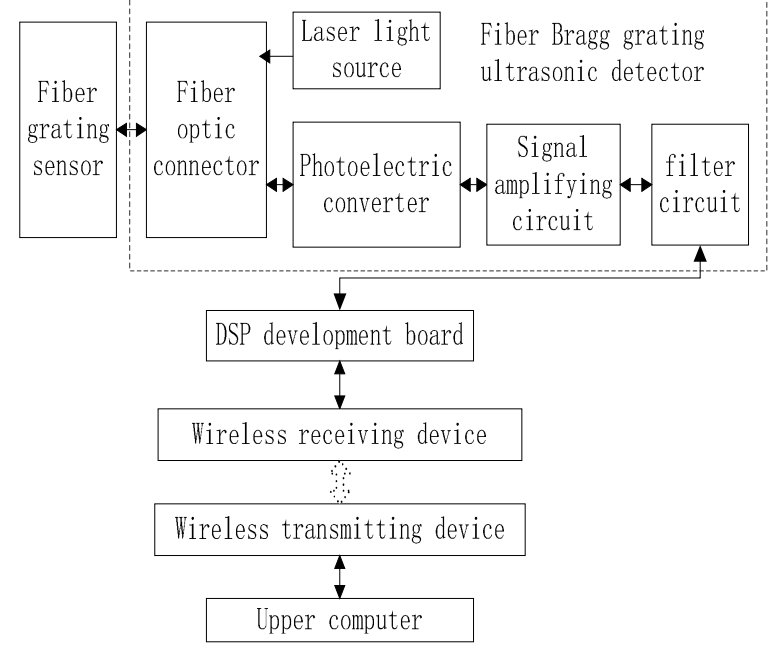

Fig.5 Frame diagram of AE signals acquisition

Considering synthetically, FBG is chosen as a high sensitivity sensor to detect AE signals. The sensing principle is that the light propagating in the fiber core will scatter at each grating surface, if the Bragg condition is satisfied, the reflected light of each grating plane is gradually accumulated, and finally a reflection peak is formed in the reverse direction, besides the central wavelength is determined by fiber parameters. FBG is essentially a narrowband filter, so the light in very narrow band is reflected to go, while the other light is transmitted out. Fig. 4 is a schematic diagram of FBG.

In this paper, the fiber Bragg grating ultrasonic detector is adopted, based on narrow linewidth tunable lasers, which can detect AE signals by the FBG sensors and send them to the processor. The FBG has a wavelength of $1553 \mathrm{~nm}$. Considering the characteristics of AE signals under crop disease stress [6], TMS320F28335 processing chip is adopted to design the acquisition and processing system of crop AE. The AE sensor is fixed on the detected plant, and the AE signal is amplified and processed into the DSP processor. The signal acquisition block diagram is shown in figure 5 . The high frequency bands of AE signals are selected, in order to avoid the influence of environmental noise. Besides the SZ05 module selected by the system has limited real-time transmission speed. Therefore, a series of characteristic parameters are extracted for AE signals in DSP processor, including amplitude, cycle, rise time, duration, threshold, etc. TMS320F28335 transfers the processed data mentioned above to the level conversion chip MAX232 through two ports of GPIO62 and GPIO63 for level conversion. The MAX232 R2IN and T2OUT two ports are connected to the RX2 and TX2 pins of the wireless module SZ05. After SZ05 wireless transmission, the SZ05 module of coordinator node receives the data and sends it to the upper computer software for real-time data storage and analysis.

\section{Host Computer Monitoring Platform}

Based on the design idea of "modularization", the monitoring system of PC is developed on the platform of LabVIEW, the design interface is shown in Figure 6. The whole software system is mainly composed of data acquisition and display module, parameter setting module, data analysis and management module, disease prevention module and so on. Among them, the data acquisition 
module is the most important part, the acquisition data of environment factor and AE signals are realized by serial port and VISA module with LabVIEW. Computer serial port communication protocol package format is shown in table 1 .

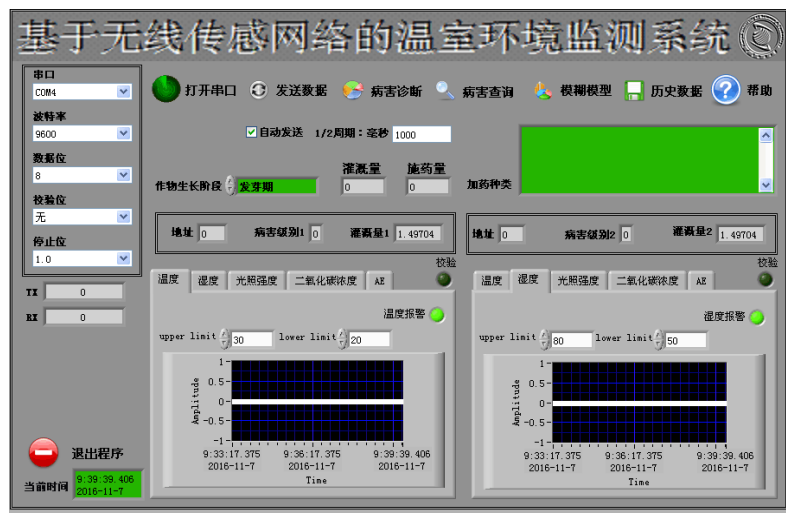

Fig.6 The host computer software

Tab.1 Data frame format of serial communication (Byte)

\begin{tabular}{cccccc}
\hline Packet header & Command code & Address bit & data & CRC parity bit & Packet tail \\
\hline 1 & 1 & 1 & $0-18$ & 2 & 1
\end{tabular}

When the host computer receives the data packet, the collected environmental parameters are saved and displayed through the waveform chart, and system determines whether the data is out of bounds and whether an alarm should be made. The control interface can also display the current collection value, collection number, accumulation value, average value, maximum value and minimum value of an environmental parameter. Environmental parameters required by different crop requirements are different. The same crop has different requirements for environmental parameters at different stages of growth. Therefore, the alarm parameters should be adjusted in time to better meet the environment required for crop growth.

\section{Conclusions}

In this paper, considering environmental parameters and AE signals under crop disease stress, a wireless monitoring system for greenhouse was proposed based on wireless sensor network technology, AE technology and virtual instrument technology. The AE characteristic was extracted, analyzed and transmitted, which improved the transmission efficiency of AE signal. To evaluate the degree of crop diseases by using AE signals, and to better serve the greenhouse monitoring. This method can also be applied to other crop disease monitoring, providing the basis for disease prevention and control.

\section{Acknowledgements}

This work was financially supported by Tianjin Research Program of Application Foundation and Advanced Technology (14JCZDJC39000).

\section{References}

[1] R.T.Wang, F.Yang: Journal of Northeast Agricultural University Vol.40(2009),p.108.

[2] X.Q.Wang, G.D.You, S.F.Yang: Transactions of the CSAE Vol.27(2011),p.205

[3] J.S.Yang, Z.H.Tian: Journal of Tianjin University of Science and Technology Vol.24(2009),p.51

[4] X.F.Zheng, G.Liu: Chinese Journal of Electron Devices Vol.37(2014),p.1189

[5] N.Li, P.Wei, H.Mo Hong, et al: Journal of Vibration and Shock Vol.34(2015),p.172

[6] X.Q.Wang, H.Y.Wang, S.F.Yang: Transactions of the Chinese Society for Agricultural Machinery Vol.45(2014),p.256 